\title{
Factores de riesgo para hidatidosis en estudiantes de enseñanza media de la comuna de Punitaqui, Chile
}

\author{
Jorge I. Gajardo y Mauricio J. Castillo
}

\section{Risk factors for hydatid disease in high school students in the district of Punitaqui, Chile}

Background: Hydatidosis is an endemic zoonosis in different areas of Chile, including the Region of Coquimbo, this is linked to the slaughter of cattle and presence of dogs. Objective: To identify risk factors related to hydatidosis in the home of high school students of Punitaqui. Material: A descriptive cross-sectional study was done by the application of a self-administered questionnaire to 274 students of high school. The information was analyzed by urban/rural area in five dimensions: knowledge, basic sanitation, keeping dogs, vegetable growing and cattle slaughtering and possession. Results: $84.6 \%$ of urban students reported not having received hydatidosis education. In rural areas, $93.5 \%$ reported owning dogs, with an average of $2.95 \mathrm{dogs} / \mathrm{home}(\mathrm{p}<0.001)$. In urban areas $44.4 \%$ of the dogs had not been dewormed and $60.3 \%(\mathrm{p}<0.001)$ was fed with viscera in rural areas. The $66.2 \%$ of cattle were goats and sheep and $64.5 \%(\mathrm{p}<0.001)$ of rural households did slaught. The main way to eliminate viscera was feeding the dogs. Conclusions: Risk factors that favor the presence of the parasite were present in relation to keeping dogs and cattle breeding and slaughter, which occurred mostly in rural areas, but being also present in urban areas.

Key words: Hydatidosis; practices; attitudes; urban and rural population; school age.

Palabras clave: Hidatidosis; prácticas; conductas; población urbana y rural; población en edad escolar.

\section{Introducción}

\section{L} a echinococcosis humana es una enfermedad zoonótica causada por las formas larvales del céstodo del género Echinococcus, el que en su estado adulto se encuentra en el intestino delgado de diferentes carnívoros como huésped definitivo, principalmente en perros. Existen variadas especies de Echinococcus, de las cuales E. granulosus (echinococosis cística) y E. multilocularis (echinococosis alveolar) son patogénicas para los humanos. El ciclo se perpetúa cuando los hospedadores definitivos se infectan al consumir vísceras que contienen la fase larval del parásito ${ }^{1,2}$.

La hidatidosis, como patología endémica, está ligada a hábitos y costumbres ancestrales difíciles de erradicar; su ciclo parasitario se favorece a través de actitudes incorporadas al acervo cultural y cuyas prácticas conducen a la transmisión de la enfermedad ${ }^{3}$.

Echinococcus granulosus, es la especie que reviste mayor importancia en salud pública, principalmente mediante el ciclo perro/ovino, en las regiones donde la enfermedad es endémica, pudiendo también intervenir otros rumiantes y suinos ${ }^{2}$. Algunas conductas y/o prácticas como el poseer gran cantidad de perros, criar ganado y alimentar perros con vísceras crudas, se consideran im- portantes factores de riesgo para padecer esta patología ${ }^{4}$.

Entre las principales medidas para combatir la hidatidosis se encuentran la desparasitación de perros, educación sanitaria, tenencia responsable de mascotas, diagnóstico y tratamiento temprano de los afectados y seguimiento epidemiológico de la enfermedad ${ }^{2}$.

Actualmente, y a pesar de las medidas de control aplicadas en algunos países, continúan apareciendo nuevos casos de hidatidosis, confirmando la vigencia que tiene la enfermedad; Chile, Argentina, Uruguay y Perú son los más afectados del cono sur ${ }^{2}$. La discapacidad laboral temporal o permanente, el aumento de gastos médicos (hasta US\$ 6.700 por paciente) $)^{5-7}$, la mayor demanda de los sistemas de salud y su gran impacto social, son sus efectos más importantes ${ }^{8,9}$.

En Chile, la hidatidosis humana es una enfermedad de notificación obligatoria (ENO) y de periodicidad diaria, de acuerdo al decreto $\mathrm{N}^{\mathrm{o}} 158$ del año 2005 del Ministerio de Salud $^{10}$. Entre los años 1990 y 2005, las tasas de incidencia de hidatidosis se mantuvieron estables y cercanas a 2 por 100 mil hbts. A contar del año 2006 hasta el 2010, hubo un paulatino descenso, con una tasa mínima de 1,41 por 100 mil hbts. el año 2010. A partir de ese año, se observa un aumento en el número de casos anuales, alcanzando una tasa de 1,81 por 100 mil hbts en el año el 2015, afectando
Universidad Católica del Norte, Coquimbo, Chile. Facultad de Medicina. Departamento de Salud Pública (JIG, MJC).

No existe relación financiera o personal con personas $u$ organizaciones que pudiesen dar lugar a conflictos de interés con este artículo. El estudio se realizó sin aportes económicos o becas.

Recibido: 2 de diciembre de 2016 Aceptado: 19 de mayo de 2017

Correspondencia a:

Mauricio Castillo Montes mcmontes@ucn.cl 
ligeramente más a hombres (52,1\%) que mujeres, y en la Región de Coquimbo para el mismo año, la tasa fue de 3,50 x 100 mil hbts afectando levemente más a mujeres $^{11-13}$. En la comuna de Punitaqui se registraron tasas de incidencia muy superiores, llegando a 38,29 (2010), 28,30 (2012) y 27,91 (2014) por $100 \mathrm{mil} \mathrm{hbts}^{14}$.

En el ámbito pecuario, la hidatidosis es la segunda causa de decomisos de órganos parasitados en mataderos en Chile por detrás de la distomatosis, representando cerca de $29 \%$ de las causas de decomisos y afectando a más de 203.000 cabezas de ganado ${ }^{15}$.

En la Región de Coquimbo la hidatidosis es una enfermedad endémica, debido principalmente a la existencia de una población rural estimada en $19,8 \%{ }^{16}$ y gran cantidad de ganado caprino (57,3\% del total nacional) y ovino. Ambos tipos de ganado son explotados de manera artesanal, principalmente por la agricultura familiar campesina. Se suma a lo anterior, altos niveles de pobreza en zonas rurales, factores que están directamente relacionados a la persistencia, emergencia y re-emergencia de la enfermedad ${ }^{8,17}$. En la provincia de Limarí se concentra $50,2 \%$ del total regional de ganado caprino, equivalente a $28,8 \%$ del total nacional ${ }^{17}$ y sólo existe un centro de faena animal para auto-consumo ubicado en la comuna de Combarbalá, el que proporciona estadísticas de decomisos con baja cantidad de sacrificio mensual. En el año 2015, este centro informó que $37,5 \%$ de los ovinos y $6,3 \%$ de los caprinos beneficiados presentaron al menos un órgano con presencia de quistes hidatídicos ${ }^{18}$.

Al suroeste de la capital provincial, Ovalle, se encuentra la comuna de Punitaqui ${ }^{19}$, la que no es ajena a la realidad regional de la enfermedad. De los $10.748 \mathrm{hbts}$ que posee, dos tercios habitan en zonas rurales conformadas por 30 pequeñas localidades que presentan características socio-culturales que favorecen la presencia de esta zoono$\operatorname{sis}^{19,20}$. A pesar de que la hidatidosis afecta principalmente a adultos, los niños y adolescentes igualmente son una población de riesgo ${ }^{21,22}$ debido a las conductas propias de su edad que facilitan el contacto con el hospedador definitivo y por ende con los huevos del parásito.

La comuna de Punitaqui posee alta ruralidad, cuya proyección estimada en el año 2012 alcanzó a 46,8\% y $25,5 \%$ de pobreza según ingresos (Región de Coquimbo fue de $16,2 \%$ ), caracterizándose además por tener en el año $201342,3 \%$ de hogares con saneamiento deficitario, cifra sustancialmente mayor a la regional $(18,6 \%)^{23-25}$. A lo anterior, se suma la gran cantidad de ganado caprino y características sociodemográficas que permiten que la enfermedad se perpetúe en el tiempo con elevada morbilidad por hidatidosis ${ }^{12,26,27}$.

El objetivo de este estudio fue identificar factores de riesgo prevalentes asociados a hidatidosis en estudiantes de enseñanza media de la comuna de Punitaqui, según lugar de residencia urbano/rural.

\section{Métodos}

\section{Diseño de estudio}

Se desarrolló un estudio descriptivo, transversal, mediante la aplicación de un cuestionario en octubre de 2014, en los únicos dos establecimientos educacionales de enseñanza media de la comuna de Punitaqui; Colegio Irma Salas Silva (CISS) y Liceo Alberto Gallardo Lorca (LAGL), de carácter particular subvencionado y municipal, respectivamente. El 54,74\% de los alumnos encuestados tenían su residencia en sectores urbanos.

\section{Instrumento}

Se utilizó un cuestionario auto-administrado anónimo en 274 estudiantes de $1^{\circ} \mathrm{a} 4^{\circ}$ año de enseñanza media, presentes el día de la aplicación (n: 78 de 92 matriculados en CISS y n: 196 de 269 matriculados en LAGL). El cuestionario constaba de 29 preguntas de selección múltiple y preguntas abiertas de respuestas cortas, teniendo todas las preguntas igual valoración (Anexo 1). Se abordaron cinco dimensiones; educación sobre hidatidosis; disponibilidad de agua potable y recolección de residuos domiciliarios; tenencia de perros, conductas y prácticas asociadas; cultivo de vegetales; tenencia y faena de ganado. El instrumento fue previamente validado en su contenido por siete expertos (tres profesores de enseñanza media, tres médicos veterinarios y una enfermera).

El estudio fue aprobado por el Comité Ético Científico de la Facultad de Medicina de la Universidad Católica del Norte, mediante Resolución del 8 de septiembre de 2014. Se requirió el consentimiento informado escrito a padres y/o apoderados y asentimiento informado de estudiantes participantes, además de la autorización de los directores de los establecimientos.

\section{Análisis estadístico}

Los datos se analizaron utilizando Microsoft Excel ${ }^{\circledR}$ y Xlstat 2011.2.06. Se agruparon de acuerdo al lugar de residencia de los alumnos (urbano o rural). Para cuantificar si las diferencias encontradas eran estadísticamente significativas, se utilizó la prueba de bondad de ajuste y la prueba de asociación de dos variables (tabla de contingencia y $\mathrm{t}$ de Student), considerando significativo un valor de $\mathrm{p}<0,05$.

\section{Resultados}

El promedio de edad de los encuestados fue de 15,9 años $\pm 1,22$, siendo mayoritario el sexo masculino $(54,74 \%)$ y la procedencia urbana $(51,45 \%)$. La mayor cantidad de alumnos cursaba $1^{\circ}$ año medio $(35,4 \%)$. 
Al consultar si habían recibido educación previa sobre hidatidosis, 79,8\% de los alumnos del sector rural, y $84,6 \%$ del sector urbano respondieron negativamente.

En la Tabla 1, se resumen las características de saneamiento básico de los hogares de procedencia, apreciándose menor cobertura de servicios en el área rural.

La mayor parte de las viviendas $(77,06 \%)$ poseía entre uno y tres perros, aunque algunas registraron ocho o más $(2,59 \%)$. Las dimensiones relacionadas a la tenencia de perros, con prácticas y conductas asociadas, se presentan en la Tabla 2. El promedio de tenencia fue de 2,9 perros por hogar, concentrada principalmente en el sector rural, con un alto número de ejemplares sin controles médicos veterinarios y desparasitaciones. El permitir el ingreso de perros al domicilio fue una práctica recurrente; sin embargo, su pernoctación al interior de las casas fue reconocida en menor frecuencia. La recogida de heces fue más frecuente en el sector urbano y la alimentación de perros con vísceras fue mayor en el sector rural, ambas con diferencias significativas. No obstante, la ingesta de vísceras con evidencias de quistes hidatídicos, fue reconocida por un bajo número de estudiantes de ambos sectores.

En la Tabla 3 se observa que mayoritariamente las madres de los alumnos realizaban la recolección de heces de perros, destacando en el sector rural el alto número de estudiantes que realizaban esta labor $(30,4 \%)$.

Acariciar perros fue una conducta común en los estudiantes, $88,7 \%$ en el sector rural y $88,0 \%$ urbano. Mientras que el dejarse lamer demostró menos aceptación en ambas zonas; $26,6 \%$ en rural versus $34,6 \%$ en el área urbana; estas diferencias no fueron significativas $(\mathrm{p}=0,357)$. Sobre $68,4 \%$ indicó lavar sus manos cinco o más veces al día y respecto al lavado posterior a acariciar perros, sobre $63,6 \%$ reconoció realizarlo siempre.

En la dimensión cultivo de vegetales, 75,0\% de los estudiantes rurales afirmó poseer huertas, frente a $48,6 \%$ de los urbanos $(\mathrm{p}<0,001)$. Respecto al tipo de vegetales cultivados, $58,1 \%$ afirmó poseer árboles frutales y 33,9\% hortalizas. El 53,19\% de los alumnos rurales y 50,68\% de urbanos manifestaron que sus perros ingresaban a las huertas.

Como se detalla en la Tabla 4, las dimensiones con mayores frecuencias en zonas rurales fueron: la tenencia de ganado $(49,2 \%)$, el sacrificio animal domiciliario $(64,5 \%)$ y realizar faena animal cinco o más veces por año $(56,3 \%)$, todas con diferencias significativas. El ganado caprino y ovino fueron los predominantes en los hogares de los encuestados, tanto en tenencia como en su faena. Las principales formas de eliminación de vísceras demostraron ser las consideradas de alto riesgo, como son la alimentación de perros $(40,7 \%)$ y la disposición en la basura $(36,4 \%)$.

\begin{tabular}{|c|c|c|c|c|c|c|}
\hline & \multicolumn{2}{|c|}{$\begin{array}{c}\text { Hogares con agua } \\
\text { potable }\end{array}$} & \multicolumn{4}{|c|}{$\begin{array}{l}\text { Hogares con } \\
\text { recolección de basura }\end{array}$} \\
\hline & $n$ & $\%$ & $p$ & $n$ & $\%$ & $p$ \\
\hline Urbano & 146 & 97,3 & \multirow[t]{2}{*}{$<0,001$} & 147 & 98,0 & \multirow[t]{2}{*}{$<0,001$} \\
\hline Rural & 65 & 52,4 & & 68 & 54,8 & \\
\hline
\end{tabular}

Tabla 2. Prácticas y conductas relacionadas a la tenencia de perros

\begin{tabular}{|c|c|c|c|c|c|}
\hline & \multicolumn{2}{|c|}{ Urbano } & \multicolumn{2}{|c|}{ Rural } & \multirow[b]{2}{*}{$\mathbf{p}$} \\
\hline & $\mathbf{n}$ & $\%$ & $\mathbf{n}$ & $\%$ & \\
\hline Hogares con perros & 115 & 76,7 & 116 & 93,6 & $<0,001$ \\
\hline Promedio perros por hogar & \multicolumn{2}{|c|}{1,6} & \multicolumn{2}{|c|}{2,9} & $<0,001$ \\
\hline Ingreso de perros a vivienda & 77 & 66,9 & 78 & 67,2 & 0,925 \\
\hline Perros duermen en vivienda & 21 & 18,3 & 20 & 17,2 & 0,976 \\
\hline Recolección de fecas & 102 & 88,7 & 72 & 62,1 & $<0,001$ \\
\hline Sin consulta veterinaria & 60 & 52,2 & 74 & 63,8 & 0,098 \\
\hline Sin desparasitar & 51 & 44,4 & 49 & 42,2 & 0,919 \\
\hline Alimentados con vísceras & 40 & 34,8 & 70 & 60,3 & $<0,001$ \\
\hline Alimentado con vísceras con quistes hidatídicos & 5 & 4,4 & 12 & 10,5 & 0,130 \\
\hline
\end{tabular}

Tabla 3. Recolección de fecas de perros en el hogar

\begin{tabular}{|lccccc|}
\hline & \multicolumn{2}{c}{ Urbano } & \multicolumn{2}{c|}{ Rural } & \\
& $\mathbf{n}$ & $\mathbf{0}$ & $\mathbf{n}$ & $\%$ & $\mathbf{p}$ \\
Madre & 31 & 34,8 & 30 & 43,5 & 0,064 \\
Padre & 14 & 15,7 & 6 & 8,7 & \\
\hline Ambos padres & 9 & 10,1 & 6 & 8,7 & \\
Otros familiares & 19 & 21,4 & 6 & 8,7 \\
Alumnos & 16 & 18,0 & 21 & 30,4 \\
\hline
\end{tabular}

\section{Discusión}

Destaca en este estudio, el alto número de estudiantes sin educación sobre hidatidosis, pese a habitar en una zona de alta prevalencia de la enfermedad ${ }^{11}$. La existencia de algunos estudiantes (principalmente en zonas rurales) que manifestaron haber recibido educación sobre la enfermedad, se podría explicar por la puesta en marcha durante el año 2010 de un programa de control y prevención de hidatidosis a pequeña escala en 13 localidades rurales de Punitaqui, por parte de la Secretaría Regional Ministerial (SEREMI) de Salud, Región de Coquimbo ${ }^{8}$.

La carencia de agua potable (beber agua de pozo o canal) y la deficiente recolección de basura domiciliaria, favorecen la transmisión de hidatidosis. Ambos servicios se encontraban disponibles en bajo porcentaje en los domicilios de estudiantes rurales, coincidiendo con la 


\begin{tabular}{|c|c|c|c|c|c|}
\hline & \multicolumn{2}{|c|}{ Urbano } & \multicolumn{2}{|c|}{ Rural } & \multirow[b]{2}{*}{$\mathbf{p}$} \\
\hline & $\mathrm{n}$ & $\%$ & $\mathbf{n}$ & $\%$ & \\
\hline \multicolumn{6}{|l|}{ Tenencia y faenamiento } \\
\hline Posee ganado & 20 & 13,3 & 61 & 49,2 & $<0,001$ \\
\hline Faenamiento domiciliario & 46 & 30,7 & 80 & 64,5 & $<0,001$ \\
\hline Faena 5 o más veces/año & 13 & 28,9 & 45 & 56,3 & 0,01 \\
\hline Perros durante faenamiento & 19 & 42,2 & 38 & 47,5 & 0,774 \\
\hline \multicolumn{6}{|l|}{ Tenencia de ganado } \\
\hline Ovino & 11 & 31,4 & 34 & 26,0 & \multirow{5}{*}{0,971} \\
\hline Caprino & 13 & 37,1 & 52 & 39,7 & \\
\hline Otros & 10 & 28,6 & 43 & 32,8 & \\
\hline $\mathrm{N} / \mathrm{R}$ & 1 & 2,9 & 2 & 1,5 & \\
\hline Total & 35 & 100 & 131 & 100 & \\
\hline \multicolumn{6}{|l|}{ Tipo de ganado faenado } \\
\hline Ovino & 26 & 34,7 & 37 & 28,5 & \multirow{5}{*}{0,723} \\
\hline Caprino & 37 & 49,3 & 73 & 56,1 & \\
\hline Otros & 10 & 13,3 & 18 & 13,9 & \\
\hline$N / R$ & 2 & 2,7 & 2 & 1,5 & \\
\hline Total & 75 & 100 & 130 & 100 & \\
\hline \multicolumn{6}{|l|}{ Forma eliminación vísceras } \\
\hline Basura & 26 & 47,3 & 33 & 30,8 & \multirow{4}{*}{0,075} \\
\hline Alimento perros & 22 & 40,0 & 44 & 41,1 & \\
\hline Enterradas & 6 & 10,9 & 21 & 19,6 & \\
\hline Quemadas & 1 & 1,8 & 9 & 8,5 & \\
\hline
\end{tabular}

población rural de Punitaqui y provincia de Limarí ${ }^{3,4,25,28}$, características comunes en países donde la enfermedad es endémica.

La tenencia de perros fue muy común en el grupo encuestado, siendo especialmente alta en zonas rurales. Esta conducta es considerada un factor de riesgo, ya que incrementa las posibilidades de padecer hidatidosis y a mayor número de perros por hogar, mayor es el riesgo de enfermar $^{29-31}$. Cifras similares a las encontradas en este estudio se describen por otros autores, tanto en sectores urbanos y rurales como en la Provincia del Limaríi ${ }^{28}$.

El ingreso de perros a las casas y dormir junto a ellos son consideradas conductas peligrosas, debido al contagio intradomiciliario que pueden generar los huevos del parásito, realidad no exclusiva de sectores rurales ${ }^{3,32,33}$. Ambas conductas fueron recurrentes en los encuestados, siendo especialmente alta la cantidad de alumnos que tenía contacto con perros.

En $37,9 \%$ de los domicilios rurales no se realizaba la recolección de residuos fecales de perros. Quienes mayoritariamente efectuaban esta labor, fueron las madres de los alumnos, destacando en el segundo lugar, los estudiantes de áreas rurales. La recolección de deposiciones sin las medidas de protección adecuadas, constituye una práctica de riesgo, ya que se tiene contacto con la fuente de eliminación de los huevos del parásito; además se ha correlacionado positivamente vivir en zonas donde no se recolectan excrementos de perros y contraer hidati$\operatorname{dosis}^{28,34}$.

Diversos autores reportan mayores prevalencias de la enfermedad en mujeres y relacionan el hecho ser mujer como un factor de riesgo ${ }^{22,33}$. A nivel nacional, esta enfermedad afecta levemente más a hombres (51,2\% año 2015), mientras que en la Región de Coquimbo, se presenta un fenómeno diferente afectando mayormente a mujeres $(56,25 \%)^{12}$, lo que podría explicarse por el mayor contacto con perros en el hogar durante las labores domésticas, que incluyen recolección de heces, y por el contacto estrecho en el uso de estos animales para arreo de ganado.

En sectores rurales y urbanos la gran mayoría de los perros nunca habían sido desparasitados ni examinados por un médico veterinario, conductas descritas como factor de riesgo de contraer echinococosis ${ }^{27,34}$.

Este estudio encontró que la alimentación de perros con vísceras es una práctica habitual en las casas de los estudiantes, comportamiento observado también en otras zonas rurales de la Provincia del Limarí. Esta conducta está calificada como la principal fuente por la cual los perros adquieren el parásito ${ }^{4,26,28}$. A lo anterior, se debe sumar que las principales formas de eliminación de vísceras fueron la alimentación de perros y eliminación en la basura domiciliaria, algo ya registrado en otras zonas de 
Chile $^{35}$. De esta manera, el acceso al consumo de vísceras puede ser directo o indirecto (a través de los residuos domiciliarios), lo que se ve favorecido por la inadecuada recolección de basura, principalmente en zonas rurales.

El alto número de estudiantes que manifestó que sus canes alguna vez habían sido alimentados con vísceras, contrasta con la baja proporción que reconoció afirmativamente alimentarlos con vísceras infectadas con quistes hidatídicos. Lo anterior podría explicarse por el desconocimiento o dificultad en reconocer quistes hidatídicos y vísceras parasitadas. Dicho desconocimiento obstaculiza a las personas reconocer la alimentación de perros con vísceras como una acción peligrosa, manteniéndose a lo largo del tiempo y arraigada en la población.

El contacto estrecho con perros es una conducta riesgosa, especialmente en niños, práctica común encontrada en este estudio ${ }^{28,36}$. El ser lamido por perros fue una conducta mayormente común en zonas urbanas, lo que se puede explicar por el rol de mascotas que cumplen estos animales, existiendo un contacto más estrecho con las personas, a diferencia de zonas rurales en donde son más utilizados para labores de trabajo como el arreo de ganado ${ }^{32}$.

El lavado frecuente de manos es un factor protector ${ }^{36}$. En este estudio el porcentaje de estudiantes que manifestaron lavarse las manos cinco o más veces al día y siempre después de acariciar un perro fue alto, demostrando así una conducta arraigada que es inculcada desde pequeños y la asumen como un acto beneficioso. Por otro lado, pudo constituir un sesgo de medición, al evitar manifestar que incurrían en una acción indebida.

El cultivo de vegetales y el acceso de perros a estas áreas, incrementan las posibilidades de transmisión de hidatidosis, por el eventual depósito de huevos del parásito en los vegetales ${ }^{28,32}$. Ambas dimensiones estuvieron presentes en un alto porcentaje de los hogares de los estudiantes encuestados.

En los domicilios, tanto rurales como urbanos, fue común la faena de ganado junto a la presencia de perros. Este comportamiento está descrito como el principal factor de riesgo siendo reportado por diferentes autores como una práctica muy arraigada en diferentes zonas de Chile $27,28,35,37$. El alto número de estudiantes urbanos que manifestó que en sus hogares se faenaba ganado (práctica más habitual en zonas rurales), se podría explicar por la migración rural-urbana y el arraigo y permanencia en el tiempo de estas costumbres s,27,32,38. $^{4}$.

La tenencia y faena mayoritaria de ganado caprino en los hogares de Punitaqui se relaciona con la gran masa ganadera de esta especie en la Región de Coquimbo, la que concentra el mayor número de cabezas de Chile ${ }^{17}$. Destaca, en segundo lugar, de importancia, el ganado ovino, y su tenencia también es un importante factor de riesgo de enfermar de hidatidosis ${ }^{4,26}$. Se suma a lo anterior, que este tipo de ganado ocupa el segundo lugar en el país en hallazgos de quistes hidatídicos en plantas de faena de animales, lo que puede interpretarse como que poseen una alta carga parasitaria ${ }^{17}$. En la Región de Coquimbo, el sacrificio de caprinos y ovinos se realiza principalmente de manera informal en los domicilios, sin ninguna supervisión médico veterinaria y sin las medidas de higiene adecuadas, lo que incrementa el riesgo ${ }^{15}$. En Chile, los genotipos de E. granulosus que más contribuyen a la hidatidosis humana son G1, G2 y G3, los que tienen como hospedador intermediario al ganado ovino, y el genotipo G6 que posee como hospedadores intermediarios a caprinos y ovinos. A lo anteriormente señalado se debe sumar que ambos tipos de ganado representan $39,3 \%$ de la masa ganadera nacional, por lo cual el riesgo de la presencia de la enfermedad es alto ${ }^{17,39}$.

En los hogares de estudiantes de enseñanza media de la comuna de Punitaqui, se presentaron diversas actitudes y prácticas que están catalogadas como factores de riesgo de producir hidatidosis, relacionadas principalmente con la tenencia de perros y la crianza y faena domiciliaria de ganado; basado en estos antecedentes se puede inferir que en la comuna el riesgo de padecer hidatidosis es alto tal como queda demostrado en las altas tasas de incidencia.

Los resultados obtenidos en este estudio, pueden extrapolarse al resto de la población de la comuna, debido a que los estudiantes de los establecimientos intervenidos, provenían de diversos sectores, tanto urbanos, periurbanos y rurales.

Todo abordaje que se realice para combatir esta enfermedad no debe centrarse sólo en la población rural, sino que también en la urbana y periurbana, debido a que las prácticas y conductas relacionadas con la enfermedad han dejado de ser exclusivas de sectores rurales. Esta orientación fue asumida por la SEREMI de Salud Coquimbo, la que inició en el año 2014 un extenso programa de prevención y control de hidatidosis en la Provincia de Limarí, específicamente en las comunas de Punitaqui, Monte Patria y Combarbalá, financiado por el Gobierno Regional de Coquimbo y ejecutado por la Universidad Católica del Norte. Su componente principal es la educación destinada a toda la población escolar, padres y apoderados, y organizaciones sociales.

Las principales formas de transmisión y perpetuación de la enfermedad son factibles de combatir mediante medidas simples, siendo la educación un pilar fundamental para lograr cambios de prácticas y conductas que se encuentran muy arraigadas en la población.

Agradecimientos: A Filomena Rojas Bugueño, Dirctora del Colegio Irma Salas Silva de Punitaqui, a Mario Rojas Rojas, Director del Dpto. de Edicación del Ilustre Municipalidad de Punitaqui y a Victor Araya Bahamondes, Director del Liceo Alberto Gallardo Lorca de Punitaqui, por su colaboración y contribución a la realización de este estudio. 


\section{Resumen}

Introducción: La hidatidosis es una zoonosis endémica en diferentes zonas de Chile y en la Región de Coquimbo, ligada a la faena de ganado y presencia de perros. Objetivo: Identificar factores de riesgo relacionados con hidatidosis en hogares de estudiantes de enseñanza media de la comuna de Punitaqui. Material: Se realizó un estudio transversal, descriptivo, aplicándose un cuestionario auto-administrado a 274 estudiantes de enseñanza media. Se analizó según zona urbana/rural en cinco dimensiones: conocimientos, saneamiento básico, tenencia de perros, cultivo de vegetales $\mathrm{y}$, tenencia y faena de ganado. Resultados: El 84,6\% de los estudiantes urbanos manifestó no haber recibido educación sobre hidatidosis. En zonas rurales, 93,5\% señaló poseer perros, promediando 2,95 perros/vivienda $(\mathrm{p}<0,001)$ y $60,3 \%(\mathrm{p}<0,001)$ era alimentado con vísceras. En zonas urbanas, 44,4\% de los perros no había sido desparasitado. El 66,2\% del alumnado declaró poseer ganado caprino y ovino y $64,5 \%(p<0,001)$ de hogares rurales faenaba. La eliminación de vísceras era destinada principalmente para alimentación de perros. Conclusiones: Se presentaron factores de riesgo que favorecen la presencia del parásito, relacionados a la tenencia de perros y crianza y faena de ganado, los que fueron mayoritarios en sectores rurales siendo igualmente altos en zonas urbanas.

Anexo 1. Encuesta epidemiológica hidatidosis

Encuesta $\mathrm{N}^{\circ}$

Fecha_ $1 / 2014 \quad$ Colegio

Edad:_ Sexo: F/M Lugar de residencia:

Curso

1. ¿Has recibido anteriormente alguna charla o educación sobre hidatidosis? a) Sí

2. ¿De dónde se obtiene el agua para consumo en tu hogar?
a) Red pública (agua potable)
b) Pozo
c) Vertiente
d) Camión aljibe
e) Otro

3. ¿Cómo es eliminada la basura que se genera en tu hogar? (puedes marcar más de una alternativa)

a) Es retirada por camiones recolectores (llevada a vertedero municipal)

b) Es depositada en lugar abierto

c) Es quemada

d) Es enterrada

e) Otro

4. ¿Cómo eliminan las excretas (fecas y orina) generadas en tu hogar?

a) Inodoro (WC o taza) conectado a alcantarillado público

b) Inodoro (WC o taza) conectado a fosa séptica

c) Letrina sanitaria (caseta sanitaria)

d) Campo abierto

e) Otra

5. ¿El piso que rodea tu casa de qué material es? (puedes marcar más de una alternativa)

a) Cemento

b) Tierra

c) Piedras

d) Pasto

e) Otro

6. ¿Hay perros en tu casa?
a) Sí ¿cuántos ?
b) No (pasar a pregunta № 15)

7. ¿Entra (n) tu (s) perro (s) a la casa?
a) Nunca
b) Casi nunca
c) A veces
d) Casi siempre
e) Siempre

8. ¿Duerme tu (s) perro (s) dentro de la casa?
a) Nunca
b) Casi nunca
c) A veces
d) Casi siempre
e) Siempre

9. ¿Alguien de tu familia recoge las fecas (deposiciones) de tu (s) perro (s)? a) Sí ¿Quién?

b) No (pasar a pregunta No 11) (pasar a pregunta $\mathrm{N}^{\circ} 10$ )

10.¿Cómo recogen las fecas de los perros? (puedes marcar más de una alternativa)

a) Con pala y escoba

b) Con bolsa

c) Con papel

d) Con cartón

e) Otra

11. ¿Con qué frecuencia desparasitan (con pastillas) a tu (s) perro (s)?
a) Nunca
b) Cada 45 días (un mes y medio)
c) Cada 2 a 3 meses
d) Cada 4 a 5 meses
e) Cada 6 o más meses 
12. ¿Tus perros han sido examinados alguna vez por un médico veterinario?

a) Sí ¿con qué frecuencia en los últimos 12 meses?

b) No

13. ¿Han alimentado alguna vez a tus perros con vísceras (asaduras o tripas)?
a) Sí
b) No

14. ¿Han alimentado a tus perros con vísceras (asaduras o tripas) con quiste hidatídico (bolsas de agua)?
a) Sí
b) No

15.¿Con que frecuencia haces cariño a perros?
a) Nunca
b) Casi nunca
c) A veces
d) Casi siempre
e) Siempre

16.¿Dejas que algún perro te lama (te lengüeteé)?
a) Sí
b) No

17. ¿Te lavas las manos después de hacer cariño a un perro?
a) Nunca
b) Casi nunca
c) A veces
d) Casi siempre
e) Siempre

18.¿Cuántas veces al día te lavas las manos?
a) 1 vez
b) 2 veces
c) 3 veces
d) 4 veces
e) 5 o más veces

19. ¿Hay huertas (huertos) en tu casa?
a) Sí (pasar a pregunta $N^{\circ} 20$ )
b) No (pasar a pregunta No 22)

20.¿Qué plantan en esas huertas?

21.¿Perros tienen acceso a las huertas?
a) Sí
b) No

22. ¿Lavas las frutas o verduras antes de consumirlas?
a) Sí
b) No

23. ¿Se faena (se carnea) ganado en tu casa?
a) Sí (pasar a pregunta $N^{\circ} 24$ )
b) No (pasar a pregunta $N^{\circ} 28$ )

24.¿Qué tipo de ganado faenan (carnean)? (puedes marcar más de una alternativa)
a) Ovejas
b) Caballos
c) Cabras
d) Cerdos
e) Otros

25. ¿En un año cuántas veces se faena ganado en tu hogar?
a) 1 vez
b) 2 veces
c) 3 veces
d) 4 veces
e) 5 o más veces

26. ¿Cómo eliminan las vísceras (asaduras o tripas) del animal faenado? (puedes marcar más de una alternativa)
a) Basura
b) Alimentan a los perros
c) Las entierran
d) Las queman
e) Otro

27.Al faenar (carnear) animales ¿Hay perros presentes cerca del área de sacrificio (carneo)?
a) Sí
b) No

28. ¿Tienen ganado en tu casa?
a) Sí (pasar a pregunta $N^{\circ} 29$ )
b) No (terminar encuesta)

29.¿Qué tipo de ganado tienen? (puedes marcar más de una alternativa)
a) Ovejas
b) Caballos
c) Cabras
d) Cerdos
e) Otros

\section{Referencias bibliográficas}

1.- Menezes da Silva A. Human echinococcosis: a neglected disease. Gastroenterol Res Practice 2010; 2010: 583297.

2.- Salvatella R, editor. Informe de la segunda reunión del Proyecto Subregional Cono Sur de Control y Vigilancia de la Hidatidosis: Argentina, Brasil, Chile y Uruguay. Segunda Reunión del Proyecto Subregional Cono Sur de Control y Vigilancia de la Hidatidosis:
Argentina, Brasil, Chile y Uruguay, 2005 Mar 17-18; Santiago, Chile. Montevideo: OPS; 2005.

3.- Remis J A, Guarnera E A, Parra A. Impacto de la hidatidosis. Influencia de factores ambientales y socioculturales en Tucumán, Argentina. Rev Argent Radiol 2009; 73 (3): 303-12.

4.- Moro P L, Cavero C A, Tambini M, Briceño Y, Jiménez R, Cabrera L. Prácticas, conocimientos $\mathrm{y}$ actitudes sobre la hidatidosis humana en poblaciones procedentes de zonas endémicas. Rev Gastroenterol Perú 2008; 28: 43-9.

5.- Fica A, Weitzel T. Gastos hospitalarios en pacientes con fascioliasis en fase aguda, hidatidosis de tratamiento quirúrgico y neurocisticercosis en un hospital general en Chile. Rev Chilena Infectol 2014; 31(4): 40610.

6.- Larrieu E, Mercapide C, Del Carpio M, Salvitti J C, Costa M T, Romeo S, et al. Evaluation of the losses produced by hydatidosis and 
cost/benefit analysis of different strategic interventions of control in the Province of Rio Negro, Argentina. Bol Chil Parasitol 2000; 55 (1-2): 8-13.

7.- $\quad$ Torgerson P R, Carmona C, Bonifacino R. Estimating the economic effects of cystic echinococcosis: Uruguay, a developing country with upper-middle income. Ann Trop Med Parasitol 2000; 94 (7): 703-13.

8.- Ministerio de Salud de Chile. Secretaría Regional Ministerial de Salud Coquimbo. Diagnóstico Situación de Salud Región de Coquimbo Año 2011. 2012.

9.- Martínez P. Caracterización de la mortalidad por hidatidosis humana. Chile, 2000-2010. Rev Chilena Infectol 2014; 31 (1): 7-15.

10.- Ministerio de Salud de Chile. Reglamento sobre notificación de enfermedades transmisibles de declaración obligatoria. Decreto $\mathrm{N}^{\circ} 158$. Santiago, 22 de octubre de 2004.

11.- Ministerio de Salud de Chile. Vigilancia Epidemiológica Enfermedades de Notificación Obligatoria. Hidatidosis. http:/epi.minsal.cl/ vigilancia-epidemiologica/enfermedades-denotificacion-obligatoria/hidatidosis/. [Accedido el 22 de diciembre de 2014].

12.- Gobierno de Chile. Ministerio de Salud. Bases de Datos ENO. http://www.deis.cl/?page $\mathrm{id}=3784$. [Accedido el 27 de abril de 2014].

13.- Ministerio de Salud de Chile. Informe Anual Situación Epidemiológica de Hidatidosis (CIE10: B67) Chile, 2015. http://epi.minsal.cl/wpcontent/uploads/2016/07/2016.07.06_Informeanual-hidatidosis-2015.pdf. [Accedido el 13 de julio de 2016].

14.- Ministerio de Salud de Chile. Secretaría Regional Ministerial de Salud Coquimbo. Departamento de Salud Pública y Planificación Sanitaria. Número de casos incidentes y tasas de incidencia de hidatidosis (CIE 10: B67.0-B67.9) por residencia, comuna de Punitaqui y Región de Coquimbo, años estadísticos 2007-2015. [Accedido por Ley de Transparencia AO043T0000677/2 de mayo de 2017].

15.- Ministerio de Agricultura de Chile. Servicio Agrícola y Ganadero. Informe beneficios y hallazgos patológicos en mataderos nacionales 2014. Santiago, abril de 2015.

16.- Instituto Nacional de Estadísticas (INE) de Chile. Productos Estadísticos. País y Regiones por Área Urbana-Rural http://www. ine.cl/canales/chile_estadistico/familias/ demograficas_vitales.php [Accedido el 1 de mayo de 2017].

17.- Instituto Nacional de estadísticas (INE) de Chile. Productos Estadísticos.http://www.ine.cl/ canales/chile_estadistico/censos_agropecuarios/ censo_agropecuario_07_comunas.php.

18.- Ministerio de Salud de Chile. Secretaría Regional Ministerial de Salud Coquimbo. Departamento de Acción Sanitaria, Unidad de
Zoonosis y Control de Vectores. Estadísticas de Patologías detectadas en establecimientos de beneficio de reses de abasto, Centro de Faena de Autoconsumo (CFA) La Unión, Combarbalá, años 2012-2016. [Accedido por Ley de Transparencia AO043T0000698/5 de mayo de 2017].

19.- Biblioteca del Congreso Nacional de Chile. Reportes Estadísticos y Comunales 2012. http:// reportescomunales.bcn.cl/2012/index.php/ Punitaqui. [Accedido el 22 de diciembre de 2014].

20.- Instituto Nacional de Estadísticas (INE) de Chile. Productos Estadísticos.http://www. ine.cl/canales/chile_estadistico/familias/ demograficas_vitales.php. [Accedido el 22 de diciembre de 2014].

21.- Lungu V. Epidemiology of human echinococcosis in the Republic of Moldova. Sci Parasitol 2012; 13 (2): 87-91.

22.- Lorca M, Garrido M, Contreras M, Salinas P, Álvarez C, Ruiz M, et al. Seroprevalencia de hidatidosis humana en la Región de Coquimbo. Chile. Parasitol Latinoam 2006; 61: 3-4.

23.- Gobierno Regional Región de Coquimbo. Departamento de Planificación Territorial. División de Planificación y Desarrollo Regional. Minuta Comuna de Punitaqui Provincia de Limarí, octubre de 2014. https:// www.gorecoquimbo.cl/gorecoquimbo/site/ artic/20150508/asocfile/20150508084911/10 minuta_punitaqui_limar_.pdf [Accedido el 1 de mayo de 2017].

24.- Ministerio de Desarrollo Social de Chile. Encuesta CASEN 2015. Indicadores Territoriales. Serie de Datos de la Tasa de Pobreza Comunal. Estimación de la pobreza por ingresos a nivel comunal 2013. http:// observatorio.ministeriodesarrollosocial.gob. cl/documentos/Sintesis-Estimacion-de-lapobreza-por-ingresos-en-comunas-20112013(nuevametodologia)07092015.pdf. [Accedido el 1 de mayo de 2017].

25.- Ministerio de Desarrollo Social de Chile. Encuesta CASEN 2015, Indicadores Territoriales, Estadísticas Comunales: Tercera Publicación 2014. http://observatorio. ministeriodesarrollosocial.gob.cl/ indicadores/pdf/comunal_general/coquimbo/ Punitaqui_2013.pdf. [Accedido el 1 de mayo de 2017].

26.- Acosta-Jamett G, Cleaveland S, Cunningham A, Bronsvoort B, Craig P. Echinococcus granulosus infection in humans and livestock in the Coquimbo region, north-central Chile. Vet Parasitol 2010; 169 (1-2): 102-10.

27.- Acosta-Jamett G, Cleaveland S, Bronsvoort B, Cunningham A, Bradshaw H, Craig P. Echinococcus granulosus infection in domestic dogs in urban and rural areas of the Coquimbo region, north-central Chile. Vet Parasitol 2010; 169 (1-2): 117-22.
28.- Acosta-Jamett G, Weitzel T, Boufana B, Adones C, Bahamonde A, Abarca K, et al. Prevalence and risk factors for echinococcal infection in a rural area of Northern Chile: A householdbased cross-sectional study. PLoS Negl Trop Dis 2014; 8 (8): e3090.

29.- Yang YR, Sun T, Li Z, Zhang J, Teng J, Liu $\mathrm{X}$, et al. Community surveys and risk factor analysis of human alveolar and cystic echinococcosis in Ningxia Hui Autonomous Region, China. Bull World Health Organ 2006; 84 (9): 714-21.

30.- Wang Q, Qiu J, Yang W, Schantz P, Raoul F, Craig P, et al. Socioeconomic and behavior risk factors of human alveolar echinococcosis in tibetan communities in Sichuan, People's Republic of China. Am J Trop Med Hyg 2006; 74(5): 856-62.

31.- Bingham G, Budke C, Larrieu E, Del Carpio M, Mujica G, Slater M, et al. A community-based study to examine the epidemiology of human cystic echinococcosis in Rio Negro Province, Argentina. Acta Trop 2014; 136: 81-8.

32.- Núñez E, Calero D, Estares L, Morales A. Prevalencia y factores de riesgo de hidatidosis en población general del distrito de NinacacaPasco, Perú 2001. An Fac Med Lima 2003; 64 (1): 34-42.

33.- Wang Q, Huang Y, Huang L, Yu W, He W, Zhong B, et al. Review of risk factors for human echinococcosis prevalence on the Qinghai-Tibet Plateau, China: a prospective for control options. Infect Dis Poverty 2014; 3 (1): 3.

34.- Escobedo C, Cubas P, Martel W. Prevalencia de Echinococcus granulosus como factor de riesgo de hidatidosis en ovinos. Investig Valdizana 2012; 6 (1): 13-6.

35.- Apt W, Pérez C, Galdámez E, Campano S, Vega F, Vargas D, et al. Equinococosis/hidatidosis en la VII Región de Chile: diagnóstico e intervención educativa. Rev Panam Salud Publica 2000; 7 (1): 8-16.

36.- Luke N, Francis E, Micheal L O, Clovice K, Ludwig S, Micheal O. A survey of potential risk factors associated with cystic echinococcosis in pastoral communities en Kasese district, Uganda. Adv Trop Med Pub Health Int 2013; 3 (1): 10-24.

37.- Santivañez S, Naquira C, Gavidia C, Tello L, Hernández E, Brunetti E, et al. Factores domiciliarios asociados con la presencia de hidatidosis humana en tres comunidades rurales de Junín, Perú. Rev Peru Med Exp Salud Publica 2010; 27 (4): 498-505.

38.- Guarnera E. Hidatidosis en Argentina: carga de enfermedad. $1^{\mathrm{a}}$ ed. Buenos Aires: Organización Panamericana de la salud; 2009.

39.- Álvarez Rojas C, Romig T, Lightowlers M. Echinococcus granulosus sensu lato genotypes infecting humans-review of current knowledge. Int J Parasitol 2014; 44: 9-18. 